\title{
Cryo-EM Reveals IMP Dehydrogenase I Filaments Adopt Diverse Architectures
}

\author{
Anika L. Burrell ${ }^{1}$, Meerit Said ${ }^{1}$, Chuankai Nie ${ }^{1}$, Matthew C. Johnson ${ }^{1}$ and Justin M. Kollman ${ }^{*}$ \\ 1. Department of Biochemistry, University of Washington, Seattle, WA, USA. \\ * Corresponding author: jkoll@uw.edu
}

Dynamic assembly of metabolic enzymes provides an evolutionarily conserved mechanism for the physical organization of cellular metabolism. This phenomenon is common in highly-regulated metabolic enzymes and has led to an emerging field aimed at understanding the physical organization of cellular metabolism. Metabolic ultrastructures have been found in diverse pathways including nucleotide biosynthesis [1], glycolysis [2], amino acid metabolism [3], and fatty acid synthesis [4]. The ultrastructures dynamically assemble and disassemble in response to changing metabolic demand, drug treatment, or stress. For many of these assemblies, the structure and biological role has remained elusive.

IMP dehydrogenase (IMPDH), a highly-conserved enzyme in purine biosynthesis, catalyzes the first committed step in GTP synthesis, and forms dynamic filamentous polymers in vertebrate cells $[5,6]$. Although IMPDH is an essential enzyme in purine biosynthesis and ultrastructures have been observed in diverse situations including activated T-cells, tumors, and retina, the role of IMPDH filaments remains unknown [7]. Mutations in one of the two isoforms IMPDH1 lead to blindness in humans and it was recently demonstrated that these mutations affect the ultrastructure of the enzyme and its ability to undergo feedback inhibition by GTP [7]. Although the molecular mechanism of how changing IMPDH1 filament structure can lead to blindness is unknown, it is clear that an understanding of the wildtype filament structure and function in the cell is essential to understanding how its disruption can lead to disease. Here, we used single particle cryo-electron microscopy (cryo-EM) and biochemical assays to determine that ligand binding promotes IMPDH1 wildtype filament formation \& determines the helical architecture of the filament and IMPDH1 filament formation protects against feedback inhibition by GTP.

We present four high resolution structures $(2.7 \AA-3.5 \AA)$ of the human enzyme IMPDH1 in a filament, obtained using single particle cryo-EM that demonstrate the IMPDH1 filament can accommodate both active and inactive protomers. The IMPDH monomer can bind four ligands: the substrates IMP and $\mathrm{NAD}+$, the allosteric effector ATP drives filament formation, and the feedback inhibitor GTP that also promotes filament assembly (Fig 1). We solved a series of filament structures to determine the effect each ligand has on the structure of the protomer within the filament and how the presence of a particular ligand affects helical architecture. The reconstructions reveal that the IMPDH1 filament can make two distinct octamer-octamer interactions that result in filaments with different helical architectures (Fig 2). In three reconstructions (ATP/NAD+, ATP/GTP, ATP/GTP/IMP) the octamer-octamer interface is identical: the tyrosine 12 of the upper monomer reaches across the interface to make a single contact with a loop on the lower monomer (Fig 2A). In the fourth reconstruction (ATP/IMP/NAD+) the octamer-octamer interface is completely different: the entire $\mathrm{N}$-terminal tail of the upper monomer reaches across the interface and binds to a pocket on the lower monomer (Fig 2B). Based on these observations, we speculate that the IMPDH1 filament acts as a scaffold where binding partners can only recognize one of the two helical architectures.

References: 
[1] GN Aughey and JL Liu, Crit Rev Biochem Mol Biol 51 (2016), p. 282.

[2] BA Webb et al., (2017) J Cell Biol 216 (2017), p. 2305.

[3] I Petrovska et al., Elife 3 (2014), p. e02409.

[4] MJ Meredith and MD Lane, J Biol Chem 253 (1978), p. 3381.

[5] WC Carcamo et al., PLos One 6 (2011), p. e29690.

[6] KC Duong-Ly et al., J Cell Science 131 (2018), p. 223289.

[7] D Fernández-Justel et al., J Mol Biol 19 (2019), p. 30029.

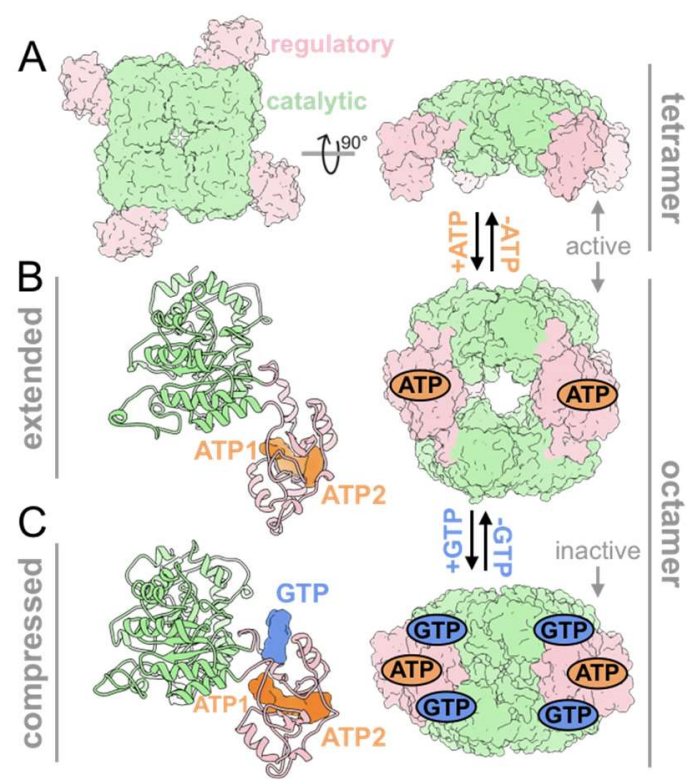

Figure 1. Nucleotide binding to the IMPDH1 regulatory domain promotes conformational changes (A) IMPDH is a tetramer in solution and each monomer has a regulatory domain (pink) and a catalytic domain (light green) (B) ATP-binding to two nucleotide binding sites in the regulatory domain stabilizes the regulatory domain. Bound ATP also promotes dimerization of regulatory domains resulting in an IMPDH octamer (C) GTP-binding in the regulatory domain locks the octamer in a compact conformation.
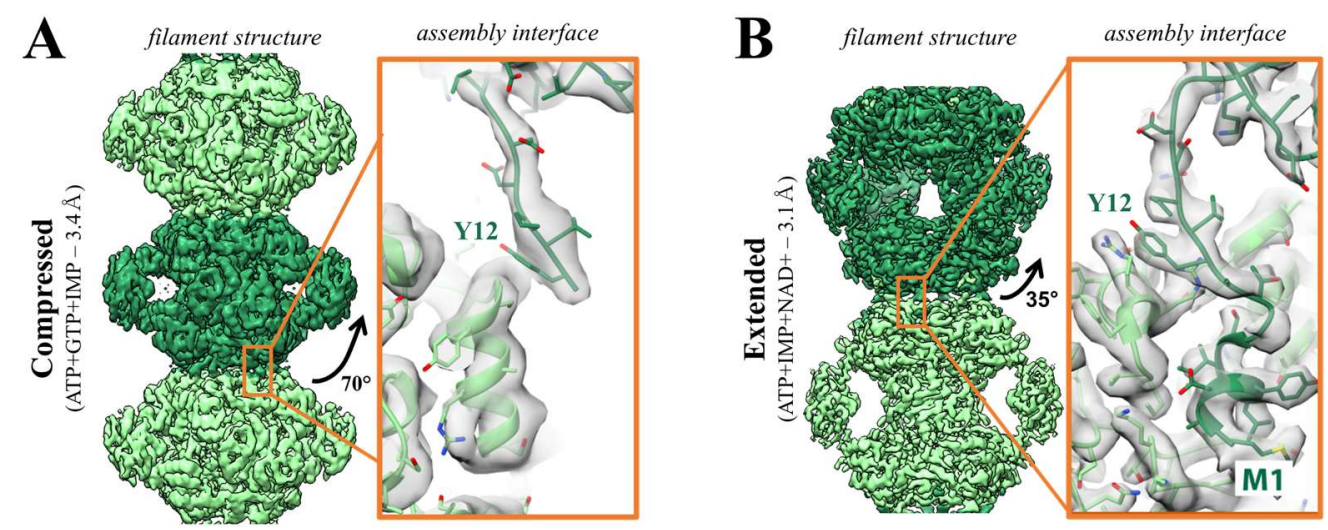

Figure 2. High resolution cryo-electron microscopy reconstructions of the IMPDH1 filament. (A) IMPDH1 compressed filament bound to ATP, GTP, \& IMP cryo-EM structure has a small assembly interface (B) IMPDH1 extended filament bound to ATP, IMP \& NAD+ has a completely different and much larger assembly interface 